\title{
Avaliação do filme lacrimal de pacientes com distonia facial durante tratamento com toxina botulínica tipo A
}

\author{
Lacrimal film evaluation of patients with facial dystonia during botulinum toxin \\ type A treatment
}

\author{
Patricia Grativol Costa ${ }^{1}$ \\ Ivana Pereira Cardoso ${ }^{2}$ \\ Fabio Petersen Saraiva ${ }^{3}$ \\ Ana Carolina Pasquini Raiza ${ }^{4}$ \\ Lílian Keiko Tanaka ${ }^{5}$ \\ Suzana Matayoshi ${ }^{6}$
}

\section{RESUMO}

Objetivo: Determinar o efeito da toxina botulínica no filme lacrimal em pacientes com distonia facial. Métodos: Foram incluídos 24 pacientes portadores de blefaroespasmo essencial e espasmo hemifacial que receberam aplicação de toxina botulínica tipo A que foram submetidos à propedêutica do filme lacrimal previamente à aplicação e após, com 7 e 30 dias. Resultados: Houve diminuição das queixas de olho seco trinta dias após a aplicação, entretanto, o tempo de ruptura do filme lacrimal e o teste de Schirmer não demonstraram variação significativa entre os períodos pré-tratamento e 1 mês da aplicação. Em relação ao teste de coloração com rosa bengala, todos os olhos que coraram no prétratamento, melhoraram na última avaliação. Conclusão: A injeção de toxina botulínica pode aliviar as queixas de olho seco nos pacientes com distonia facial pela provável ação de inibição do orbicular na sua função de bomba lacrimal.

Descritores: Drenagem; Espasmo hemifacial/terapia; Piscadela; Rosa Bengala; Toxina botulínica tipo A/uso terapêutico; Distonia

\section{INTRODUÇÃO}

As distonias faciais são caracterizadas por contrações espasmódicas involuntárias repetidas dos músculos faciais, incluindo o músculo orbicular dos olhos, resultando no fechamento palpebral prolongado. O blefaroespasmo essencial é uma distonia focal adquirida das pálpebras caracterizada por contrações tônicas espasmódicas do músculo orbicular, prócerus e corrugador dos supercílios. Acredita-se que seja uma doença neurológica provavelmente relacionada a alterações nos gânglios da base. O espasmo hemifacial é também uma distonia focal caracterizada pela contração involuntária e intermitente dos músculos da face, geralmente unilateral. A compressão vascular do nervo facial é sua etiologia mais provável ${ }^{(1-2)}$.

É relatada na literatura a associação de olho seco em pacientes com distonia facial, pois a contração do músculo orbicular periódica é fundamental para a manutenção e renovação do filme lacrimal ${ }^{(3)}$. Além disso, este músculo também é considerado o principal responsável pelo mecanismo de bomba lacrimal, auxiliando a drenagem da lágrima ${ }^{(4-5)}$.

A toxina botulínica tipo A foi introduzida para denervar quimicamente a musculatura ocular extrínseca em 1978 por $\operatorname{Scott}^{(6-7)}$. A injeção da toxina na porção medial das pálpebras pode provocar uma denervação farmacológica temporária do músculo orbicular. Dessa forma, o uso desta medicação pode estar relacionado com a diminuição da drenagem lacrimal e com o alívio dos 
sinais e sintomas de olho seco em pacientes com blefaroespasmo essencial e espasmo hemifacial ${ }^{(4)}$.

Dessa forma, o objetivo deste estudo foi determinar o efeito da toxina botulínica no filme lacrimal em pacientes com distonia facial através da propedêutica para olho seco.

\section{MÉTODOS}

Estudo prospectivo, realizado no setor de Plástica Ocular do Hospital das Clínicas da Universidade de São Paulo (HCFMUSP). Foram triados 24 pacientes, sendo que, 16 eram portadores de blefaroespasmo essencial e 8 de espasmo hemifacial. Foram submetidos à aplicação de toxina botulínica tipo $\mathrm{A}\left(\right.$ Botox $\left.^{\circledR}\right)$ na dose de 12,5 unidades ao redor de cada olho $(2,5$ unidades por ponto de aplicação; figura 1). A diluição da toxina utilizada foi de 100 unidades de Botox ${ }^{\circledR}$ em $4 \mathrm{ml}$ de cloreto de sódio a $0,9 \%$. Todos os pacientes já vinham recebendo este tipo de tratamento com intervalo de aproximadamente 6 meses entre as aplicações. Além disso, estes pacientes selecionados não fizeram uso de colírios lubrificantes antes do tratamento e durante o período de seguimento.

Foram analisados os seguintes dados: idade, sexo, tempo da doença, anamnese (questionando a respeito de sinais e sintomas relacionados com olho seco e medicações sistêmicas que pudessem interferir no filme lacrimal), e os exames de propedêutica do filme lacrimal (tempo de ruptura do filme lacrimal BUT, teste de Schirmer e coloração com Rosa Bengala).

O teste do BUT foi realizado com aplicação de colírio de fluoresceína no fundo de saco conjuntival e medida na lâmpada de fenda do tempo de ruptura do filme lacrimal. Um resultado menor que 5 segundos foi considerado patológico ${ }^{(3,8)}$.

O teste de Schirmer foi realizado com papel de filtro (Whatman 41, medindo $5 \mathrm{~mm}$ de largura por $35 \mathrm{~mm}$ de extensão), dobrando o papel $5 \mathrm{~mm}$ antes da ponta terminal e colocando no
1/3 externo da pálpebra inferior, sem aplicação de colírio anestésico. Foi medida a extensão umidecida ao final de 5 minutos. A leitura da fita com régua milimetrada menor que $5 \mathrm{~mm}$ foi considerada anormal ${ }^{(3,8)}$.

O teste da coloração com Rosa Bengala foi realizado aplicando-se 1 gota de colírio de Rosa Bengala no fundo de saco conjuntival observando-se na lâmpada de fenda a coloração de células desvitalizadas. Os resultados foram classificados em:

Grau I - coloração de pontos distribuídos na área exposta da conjuntiva bulbar e na parte inferior da córnea;

Grau II - coloração de toda a área interpalpebral;

Grau III - presença de filamentos mucosos corados na porção exposta da superfície corneana;

Grau IV - observação de grande quantidade de filamentos mucosos ${ }^{(8)}$.

A propedêutica do filme lacrimal foi realizada antes da aplicação da toxina botulínica e repetida após 1 semana e 1 mês. Os resultados foram comparados, utilizando se o teste $t$ de Student para amostras independentes, com um nível de significância menor ou igual a 0,01 (1\%), comparando o tempo de ruptura do filme lacrimal e o teste de Schirmer de todos os pacientes para cada época em que foram realizados (pré-tratamento, 1 semana e 1 mês).

\section{RESULTADOS}

Foram avaliados no estudo 40 olhos de 16 pacientes portadores de blefaroespasmo essencial ( 32 olhos) e 8 pacientes com espasmo hemifacial ( 8 olhos) com indicação de tratamento com toxina botulínica tipo A. Foi incluído apenas o olho do lado afetado nos casos de espasmo hemifacial.

O grupo era constituído de 16 pacientes do sexo feminino e 8 pacientes do sexo masculino com idade variando entre $45 \mathrm{e}$ 85 anos. A duração do espasmo foi de 3 a 30 anos, sendo que

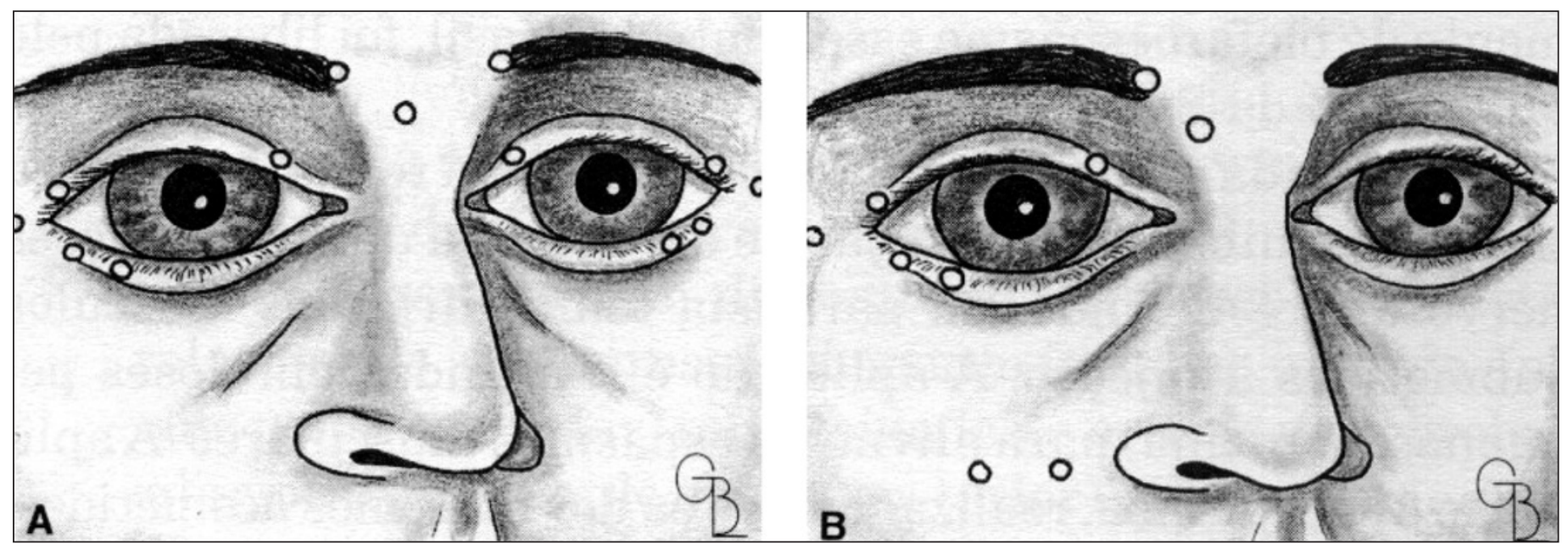

Figura 1 - Locais de aplicação da toxina botulínica no blefaroespasmo essencial (A) e no espasmo hemifacial (B).

Fonte: Matayoshi S, Siva AB. Blefaroespasmo essencial. In: Matayoshi S, Forno EA, Moura EM. Manual de cirurgia plástica ocular. São Paulo: Roca; 2004. p.127-35. Reproduzido e cedido pela autora. 
41,6\% dos pacientes apresentavam a doença há mais de 10 anos. Nenhum dos pacientes estava em uso de medicações sistêmicas que pudessem interferir na produção da lágrima.

Conforme mostra o gráfico 1, no período pré-tratamento, todos os pacientes relataram algum sintoma de olho seco, sendo os mais citados: prurido $(58,33 \%)$, sensação de corpo estranho $(50,0 \%)$ e lacrimejamento $(45,83 \%)$. Trinta dias após a injeção de toxina botulínica, houve diminuição expressiva dos sinais e sintomas, sendo que os mais citados foram: secreção $(33,33 \%)$, prurido $(29,16 \%)$ e lacrimejamento $(25,0 \%)$.

Em relação ao BUT, no pré-tratamento encontramos uma média de 6,27 segundos, com 1 semana média de 5,5 segundos e com 1 mês média de 9,2 segundos. O teste de Schirmer resultou, em média, de 13,3 mm; 10,3 $\mathrm{mm}$ e $11,5 \mathrm{~mm}$ no prétratamento, $7^{\circ}$ e $30^{\circ}$ dias pós-tratamento, respectivamente. De 40 olhos avaliados pelo teste de coloração com Rosa Bengala no pré-tratamento, 4 apresentaram resultado grau I, 2 grau II, 3 grau III e nenhum olho grau IV. Após 1 mês, todos os olhos que coraram com Rosa Bengala no pré-tratamento, tiveram exame negativado.

\section{DISCUSS ÃO}

O olho seco é uma doença da superfície ocular causada por diversas patologias que alteram o mecanismo de proteção externa ocular levando a uma instabilidade do filme lacrimal ${ }^{(3)}$. Há relatos na literatura relacionando blefaroespasmo com olho seco, porém os resultados são controversos ${ }^{(3,9)}$. Apesar de ser difícil determinar se realmente existe essa relação, sabe-se que o blefaroespasmo interrompe o ato de piscar periódico que é fundamental para a manutenção e renovação do filme lacrimal ${ }^{(3)}$.

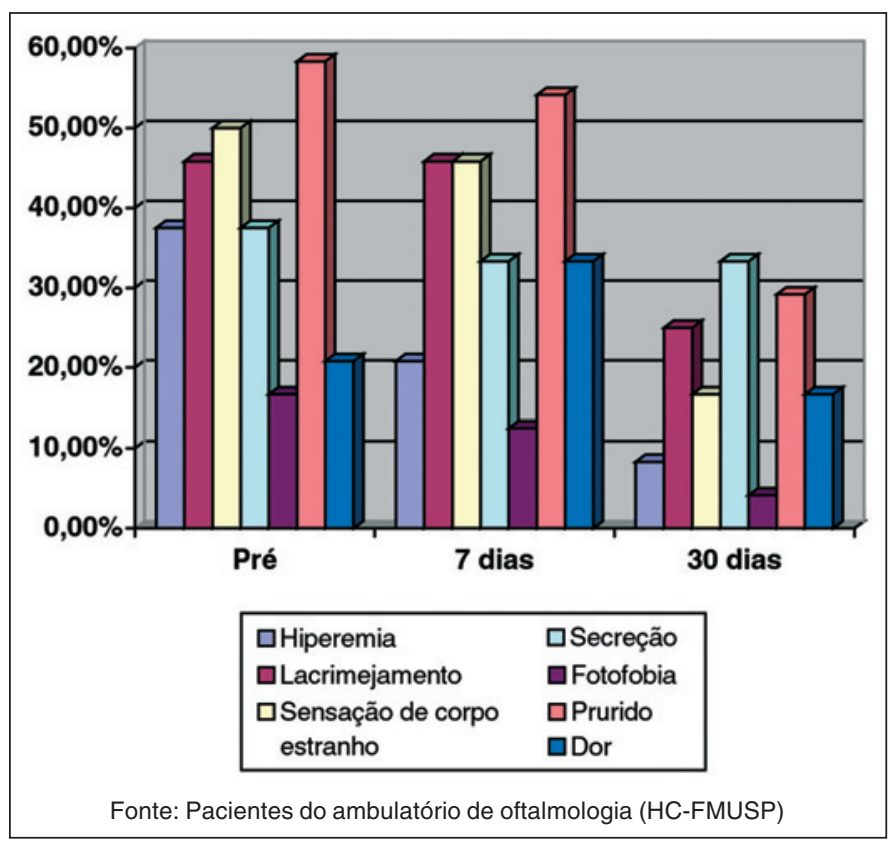

Gráfico 1 - Distribuição das queixas dos pacientes no período pré-tratamento, 7 dias pós tratamento e 30 dias pós tratamento - São Paulo - 2004
A anatomia e a fisiologia do sistema lacrimal vêm sendo estudadas e investigadas desde o século XVIII. Existe uma forte evidência de um mecanismo de bomba associado ao canalículo lacrimal e o músculo orbicular é considerado o principal responsável por este mecanismo ${ }^{(4-5)}$. A paralisia do músculo orbicular palpebral após o uso da toxina botulínica poderia diminuir a função de bomba lacrimal durante o piscar.

Este estudo investigou a influência que a injeção de toxina botulínica pode exercer no filme lacrimal. A análise dos sinais e sintomas de olho seco apresentados no gráfico 1 mostra que, de um modo geral, houve uma diminuição das queixas trinta dias após a aplicação. Entretanto, o BUT, que avalia a estabilidade do filme lacrimal, e o teste de Schirmer, que avalia a produção da camada aquosa, não demonstraram variação significativa $(\mathrm{p}<0,01)$ entre os períodos pré-tratamento, após 1 semana e após 30 dias da aplicação (Tabela 1). Em contraste com os resultados deste estudo, Horwath-Winter et al., observaram uma diminuição dos valores obtidos no teste de Schirmer. Estes autores especulam que a diminuição dos valores encontrados no teste de Schirmer possa ser devido à idade relativamente alta dos pacientes estudados ${ }^{(3)}$.

O corante de Rosa Bengala cora células em degeneração ou mortas e células saudáveis recobertas por muco e filamentos. A maioria dos pacientes (77,5\%), no pré-tratamento apresentaram o teste com coloração por Rosa Bengala negativo. Todos os olhos alterados (22,5\%) mostraram melhora após 30 dias de tratamento (Tabela 2).

Uma razão que poderia explicar os resultados encontrados no teste de BUT e de Schirmer é o fato de que a toxina botulínica pode ter um efeito farmacológico direto na produção de lágrima pela glândula lacrimal quando aplicada intraglandular, e com menor eficácia via subcutânea ${ }^{(10-11)}$. A toxina botulínica tipo A afeta a transmissão colinérgica durante as manifestações sistêmicas do botulismo. Seu uso está sendo estudado para o tratamento de patologias com hiperatividade secretória como "lágrima de crocodilo", hipersalivação e hiperidrose. A toxina botulínica tipo A pode diminuir a secreção da

\begin{tabular}{|lccc|}
\hline \multicolumn{4}{|c|}{ Tabela 1. Média dos resultados dos testes do BUT e Schirmer dos } \\
pacientes avaliados nos três períodos \\
Testes & Pré-tratamento & $\mathbf{1}$ semana & $\mathbf{1}$ mês \\
BUT $(\mathrm{s})$ & 6,27 & 5,5 & 9,2 \\
Schirmer $(\mathrm{mm})$ & 13,30 & 10,3 & 11,5 \\
Fonte: Pacientes do ambulatório de oftalmologia (HC-FMUSP) \\
Estatística: Teste t de Student $(\mathrm{p}<0,01)$
\end{tabular}

\begin{tabular}{|c|c|c|c|c|c|}
\hline $\mathrm{N}=40$ & Normal & Grau I & Grau II & Grau III & Grau IV \\
\hline Pré & 31 & 4 & 2 & 3 & 0 \\
\hline 1 semana & 35 & 5 & 0 & 0 & 0 \\
\hline 1 mês & 40 & 0 & 0 & 0 & 0 \\
\hline
\end{tabular}


glândula lacrimal através da sua dispersão local pelos tecidos, quando aplicada no músculo orbicular ${ }^{(3)}$. Logo, a droga deve ter um efeito positivo no filme lacrimal ao melhorar o blefaroespasmo e paralisar o orbicular, reduzindo a drenagem lacrimal, e por outro lado, um efeito negativo, diminuindo a produção de lágrima pela dispersão local (efeito anticolinérgico). $\mathrm{O}$ resultado no filme lacrimal provavelmente dependerá do local e forma de aplicação, dose e dispersão tecidual local da droga.

\section{CONCLUSÕES}

A injeção de toxina botulínica pode aliviar os sinais e sintomas de olho seco em pacientes com blefaroespasmo. Isso talvez esteja relacionado à inibição da ação do músculo orbicular com diminuição da drenagem lacrimal. Porém existem outros efeitos associados da toxina botulínica que precisam ser melhor estudados e que também podem influenciar na produção da lágrima. Pode representar um tratamento promissor em pacientes com olho seco e distonias faciais.

\section{ABSTRACT}

Purpose: To determine the effect of botulinum toxin injection in the eyelid on lacrimal film in patients with facial dystonia. Methods: Twenty-four patients with essential blepharospasm and hemifacial spasm were submitted to botulinum toxin injection and lacrimal film tests were performed before the application and after seven and thirty days. Results: There was improvement in symptoms of dry eye and rose bengal test, however, the breakup time and Schirmer's test did not show significant variation between pretreatment and after 1 month of follow-up. Conclusion: The dry eye symptoms in patients with facial dystonia may be attenuated by botulinum toxin due to its possible inhibitory effect on the orbicular muscle leading to a decrease in lacrimal pump.

Keywords: Drainage; Hemifacial spasm/therapy; Blinking; Rose Bengal; Botulinum toxin type A/therapeutic use; Dystonia

\section{REFERÊNCIAS}

1. Matayoshi S, Siva AB. Blefaroespasmo essencial. In: Matayoshi S, Forno EA, Moura EM. Manual de cirurgia plástica ocular. São Paulo: Roca; 2004. p.127-35.

2. Machado FCN, Fregni F, Campos CR, Limongi JCP. Espasmo hemifacial bilateral: relato de caso. Arq Neuropsiquiatr. 2003;61(1):115-8.

3. Horwath-Winter J, Bergloeff J, Floegel I, Haller-Schober EM, Schmut O. Botulinum toxin A treatment in patients suffering from blepharospasm and dry eye. Br J Ophthalmol. 2003;87(1):54-6.

4. Dutton JJ. The lacrimal drainage system. In: Dutton JJ. Atlas of clinical and surgical orbital anatomy. Philadelphia: WB Saunders; 1994. p.142-5.

5. Sahlin S, Chen E, Kaugesaar T, Almqvist H, Kjellberg K, Lennerstrand G. Effect of eyelid botulinum toxin injection on lacrimal drainage. Am J Ophthalmol. 2000;129(4):481-6.

6. Reifler DM. Early descriptions of Horner's muscle and the lacrimal pump. Surv Ophthalmol. 1996;41(2):127-34.

7. Kraft SP, Lang AE. Botulinum toxin injections in the treatment of blepharospasm, hemifacial spasm, and eyelid fasciculations. Can J Neurol Sci. 1988;15(3): 276-80.

8. Scarpi MJ. Olho seco. In: Kara-José N, Belfort Junior R. In: Córnea ClínicaCirúrgica. São Paulo:Roca;1997. p287-99.

9. Price J, O'Day J. A comparative study of tear secretion in blepharospasm and hemifacial spasm patients treated with botulinum toxin. J Clin Neuroophthalmol. 1993;13(1):67-71.

10. Hofmann RJ. Treatment of Frey's syndrome (gustatory sweating) and 'crocodile tears' (gustatory epiphora) with purified botulinum toxin. Ophthal Plast Reconstr Surg. 2000;16(4):289-91.

11. Keegan DJ, Geerling G, Lee JP, Blake G, Collin JR, Plant GT. Botulinum toxin treatment for hyperlacrimation secondary to aberrant regenerated seventh nerve palsy or salivary gland transplantation. Br J Ophthalmol. 2002;86(1): 43-6.

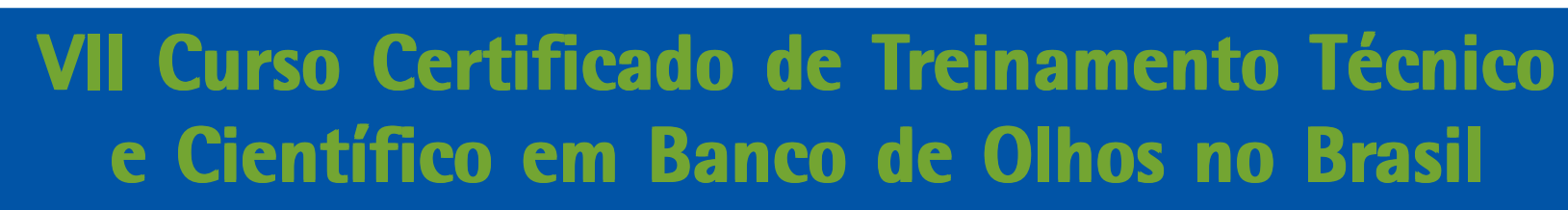

\section{3 a 29 de Julho de 2006 \\ São Paulo - SP}

INFORMAÇÕES

Tel.: (21) 2539-6345•E-mail: apabo@uninet.com.br 